\title{
Comparative Analysis of Vertebroplasty and Kyphoplasty for Osteoporotic Vertebral Compression Fractures
}

\author{
Melih Bozkurt ${ }^{1}$, Gokmen Kahilogullari ${ }^{1}$, Mevci Ozdemir ${ }^{2}$, \\ Onur Ozgural ${ }^{1}$, Ayhan Attar ${ }^{1}$, Sukru Caglar ${ }^{1}$, Can Ates ${ }^{3}$ \\ ${ }^{1}$ Department of Neurosurgery, Ankara University School of Medicine, Ankara, Turkey \\ ${ }^{2}$ Department of Neurosurgery, Pamukkale University School of Medicine, Denizli, Turkey \\ ${ }^{3}$ Department of Biostatistics, Ankara University School of Medicine, Ankara, Turkey
}

Study Design: A retrospective study.

Purpose: The aim of this study is to compare the efficacy and outcome of vertebroplasty compared with unipedicular and bipedicular kyphoplasty for the treatment of osteoporotic vertebral compression fractures in terms of pain, functional capacity and height restoration rates.

Overview of Literature: The vertebroplasty procedure was first performed in 1984 for the treatment of a hemangioma at the C2 vertebra. Kyphoplasty was first performed in 1998 and includes vertebral height restoration in addition to using inflation balloons and high-viscosity cement. Both are efficacious, safe and long-lasting procedures. However, controversy still exists about pain relief, improvement in functional capacity, quality of life and height restoration the superiority of these procedures and assessment of appropriate and specific indications of one over the other remains undefined.

Methods: Between 2004 and 2011, 296 patients suffering from osteoporotic vertebral compression fracture underwent 433 vertebroplasty and kyphoplasty procedures. Visual analogue scale (VAS), the Oswestry Disability Index (ODI) and height restoration rates were used to evaluate the results.

Results: Mean height restoration rate was $24.16 \% \pm 1.27 \%$ in the vertebroplasty group, $24.25 \% \pm 1.28 \%$ in the unipedicular kyphoplasty group and $37.05 \% \pm 1.21 \%$ in the bipedicular kyphoplasty group. VAS and ODI scores improved all of the groups.

Conclusions: Vertebroplasty and kyphoplasty are both effective in providing pain relief and improvement in functional capacity and quality of life after the procedure, but the bipedicular kyphoplasty procedure has a further advantage in terms of height restoration when compared to unipedicular kyphoplasty and vertebroplasty procedures.

Keywords: Vertebral column; Spine; Osteoporosis; Vertebral compression fractures; Kyphoplasty; Vertebroplasty

\section{Introduction}

Osteoporosis is a common health problem that can have a serious impact on the elderly population. It is characterized by low bone mass density triggering debilitated bones that are at elevated risk for fracture [1]. Conse-

Received Aug 13, 2012; Revised Sep 17, 2012; Accepted Sep 20, 2012

Corresponding author: Mevci Ozdemir

Department of Neurosurgery, Pamukkale University School of Medicine,

20070, Kinikli, Denizli, Turkey

Tel: +90-258-444-0728, Fax: +90-258-213-4922 , E-mail: drmevci@hotmail.com 
Table 1. General properties of the patients

\begin{tabular}{|c|c|c|c|c|}
\hline Characteristic & Vertebroplasty & $\begin{array}{l}\text { Unipedicular } \\
\text { kyphoplasty }\end{array}$ & $\begin{array}{l}\text { Bipedicular } \\
\text { kyphoplasty }\end{array}$ & $p$-value \\
\hline Age (yr) & $57 \pm 10$ & $57 \pm 9.4$ & $58 \pm 9.8$ & $>0.05$ \\
\hline Gender (female/male) & $50 / 46$ & $73 / 43$ & $51 / 33$ & $>0.05$ \\
\hline Mean t score & -2.45 & -2.49 & -2.48 & $>0.05$ \\
\hline Mean injury time (mo) & 22.91 & 23.88 & 23.44 & $>0.05$ \\
\hline Mean follow-up (mo) & 42.90 & 41.80 & 43.40 & $>0.05$ \\
\hline Mean VAS score (preoperative) & 8.30 & 8.30 & 8.27 & $>0.05$ \\
\hline Mean VAS score (postoperative 1 yr) & 2.78 & 2.84 & 2.66 & $>0.05^{\mathrm{al}},<0.001^{\mathrm{bl}}$ \\
\hline Mean ODI (preoperative) & 74.42 & 73.45 & 73.80 & $>0.05$ \\
\hline Mean ODI (postoperative 1 yr) & 26.56 & 26.25 & 26.01 & $>0.05^{\mathrm{a} l},<0.001^{\mathrm{bl}}$ \\
\hline Mean compression rate (\%) & 40 & 40 & 41 & $>0.05$ \\
\hline Mean height restoration rate (\%) & $24.16 \pm 1.27$ & $24.25 \pm 1.28$ & $37.05 \pm 1.21$ & $>0.05^{\mathrm{c})},<0.001^{\mathrm{d})},<0.001^{\mathrm{e})}$ \\
\hline
\end{tabular}

VAS, visual analogue scale; ODI, Oswestry Disability Index.

${ }^{a}$ Difference between groups; ${ }^{\text {bl}}$ Difference between pre and postoperative values; ${ }^{c}$ Difference between vertebroplasty and unipedicular kyphoplasty;

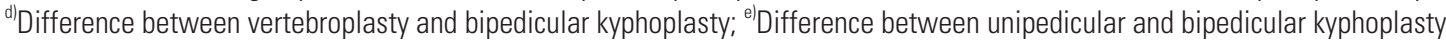

quences of osteoporosis generally include vertebral, hip, wrist, and ankle fractures [2]. The frequency of vertebral fractures increases with age. Lifetime risk of developing vertebral fracture over the age of 50 is $8 \%$ for females, while over the age of 65 , the lifetime risk is $27 \%$ for both genders [3]. Approximately 750,000 new cases of osteoporosis are observed in the United States annually [4]. Osteoporosis and related complications like pain, incapacitated motility, spinal deformity, sleep disorders, psychiatric problems, and pulmonary complications have an unfavorable influence on public health [5].

Initial therapy for osteoporotic vertebral compression fractures (OVCF) are bed rest, orthotic devices and pain medication $[1,6]$. However, some patients fail to benefit from these treatment modalities and disease-related morbidity and mortality persists. Conservatively treated OVCF's are cured with partial relief of pain and quality of life within 2 to 12 weeks $[7,8]$. Handling of OVCF's, which do not respond to noninvasive management strategies and do not lead to spinal canal compromise or neurological deficit requiring decompression and surgical instrumentation, is accomplished with two minimally invasive procedures, vertebroplasty and kyphoplasty.

Pain and disability associated with OVCF are treated effectively by augmentation of compressed vertebrae with percutaneous vertebroplasty or kyphoplasty. But there is still controversy about pain relief, improvement in functional capacity, quality of life and height restoration the superiority of these procedures and assessment of appropriate and specific indications of one over the other remains undefined. The aim of this study is to compare the efficacy and outcome of percutaneous vertebroplasty and kyphoplasty and the unipedicular versus bipedicular approach.

\section{Materials and Methods}

Between 2004 and 2011, 296 patients suffering from OVCF underwent 433 consecutive vertebroplasty and kyphoplasty procedures. General properties of the patients are summarized in Table 1.

Patients with OCVF are included and vertebroplasty/ kyphoplasty are used as an intervention in this study. Patients who included spinal cord and thecal sac compression were excluded from the study. Complete physical and neurological examinations and pain scores were verified. All patients underwent lateral and anteroposterior (AP) standing X-ray, and thin slice computerized tomography (CT) and magnetic resonance imaging (MRI) of the compressed vertebrae before surgery (Fig. 1).

Preoperative and postoperative X-rays were reviewed. Lost vertebra body height was calculated; Height of caudal or cranial adjacent vertebrae-preoperative height of the effected vertebrae/height of caudal or cranial adjacent 
vertebrae $\times 100$. After that height of restoration was calculated as; postoperative fracture height-preoperative fracture height/100-preoperative fracture height [9].

Both vertebroplasty and kyphoplasty procedures were performed under local anesthesia with sedation. Patients are placed in the prone position and two C-arm fluoroscopies, both antero-posterior and lateral, were used during the intervention to obtain biplanar images. The transpedicular route was used for all interventions. For the bipedicular kyphoplasty procedure, the Jamshidi needle was inserted in the pedicle of the fractured vertebrae. $\mathrm{K}$-wire was inserted through the needle and the needle was replaced by working cannula and biopsy was taken and the bone was drilled. After that the inflatable balloon was inserted into the vertebrae. Mean procedure time per level was 10 minutes. For vertebroplasty and unipedicular kyphoplasty procedures, the Jamshidi needle was inserted into the left side of the fractured vertebrae. Injection of the cement was observed in real time by two C-arm fluoroscopies.

Percutaneous vertebrae augmentation intervention was performed for the treatment of OVCF, in whom, 4 weeks of conservative therapy with the oral administration of non-steroidal anti-inflammatory drugs and supportive orthosis had failed. Early augmentation is indicated for the patients who had inability to mobilize and had fractures less likely improve with conservative treatment modalities such as thoracolumbar junction fractures.

The selection criteria for intervention groups were determined by the changes in the government health insurance subsidization policy. During a seven-year period only one of the three given procedures was subsidized at a given time by the government. Therefore, the procedure

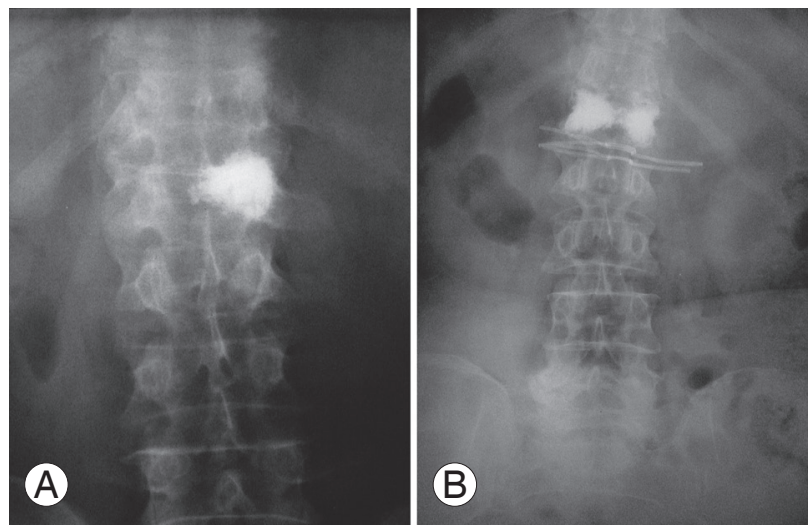

Fig. 1. (A) Anteroposterior (AP) X-ray view of unipedicular kyphoplasty. (B) AP X-ray view of bipedicular kyphoplasty. of choice was dictated by the subsidization policy at the date of admission, which in turn created three completely randomized patient groups consecutively in seven years. In our opinion this situation decreased the chance of selection bias. These groups were: bipedicular kyphoplasty, unipedicular kyphoplasty and vertebroplasty.

The decision regarding the indication between the uni and bipedicular approach was made according to the variety of the vertebral fracture individually. If unilateral pedicular impairment or pedicle asymmetry were observed, the unipedicular approach was selected. Furthermore, when the fracture delineation expanded to both halves of the vertebral body on axial computerized tomography images, the unipedicular approach was also carried out.

Age, gender, preoperative t-scores and mean injury time of the patients were analyzed. For every effected level, compression and height restoration rates and the amount of injected cement were recorded. Lateral standing X-ray was used to measure the compression and height restoration rates. Postoperative X-rays were performed one day after the surgery.

Of the 296 patients, 200 were subjected to kyphoplasty and 96 to vertebroplasty procedures. One hundred sixteen kyphoplasty procedures were performed via the unipedicular approach and 84 via the bipedicular approach. All 96 of the vertebroplasty procedures were performed via the unipedicular approach.

Pain scores were recorded for all patients using the VAS preoperatively, postoperatively, at the first day, sixth month and first year. The Oswestry Disability Index was calculated to measure patients' permanent functional disability preoperatively and postoperatively at 6 months and 1 year [10]. Statistical analysis of the VAS and ODI values and height restoration rates were performed to compare the vertebroplasty, unipedicular kyphoplasty and bipedicular kyphoplasty groups. Patients, whom multiple procedures were performed in a group, mean VAS and ODI values before and after each intervention, were calculated and statistical analyses were performed depending on these values.

Friedman two-way analysis of variance by ranks evaluated differences among four evaluation points for VAS and ODI scores. When the $p$-value from the Friedman test statistics was statistically significant, a multiple comparison test was used to know which frequency band differed from the others [11]. Comparison among tho- 
racolumbar injured vertebrae levels was assessed by the chi-square test. The groups were compared with respect to the VAS and ODI scores by nonparametric analyses of longitudinal data in factorial analysis (F1-LD-F1 design) [12]. $p$-values less than 0.05 were considered significant. SPSS Windows 11.5 and R ver. 2.13.1 (SPSS Inc., Chicago, IL, USA) were used for the statistical analyses.

\section{Results}

One hundred and thirty-seven vertebroplasties were performed in 96 patients and 296 kyphoplasties were performed in 200 patients. All of the vertebroplasty procedures were performed with unipedicular approach, while 84 kyphoplasty patients were treated with a bipedicular approach and 116 kyphoplasty patients with a unipedicular approach. 187 patients received single and 109 patients received multiple procedures. Distribution of treated levels for groups were; between T5-L5 for vertebroplasty, between T4-L5 for unipedicular kyphoplasty and between T4-L5 for bipedicular kyphoplasty.

The 296 patients ranged in age from 43 to 87 years (mean, 61.1 years; median, $57 \pm 9.6$ years). Of all patients, $180(60.8 \%)$ were female and $116(29.2 \%)$ were male. The mean $\mathrm{t}$-score was -2.48 in all patients, -2.45 in the vertebroplasty group -2.49 in unipedicular kyphoplasty group, and -2.48 in the bipedicular kyphoplasty group. The mean injury time before the intervention was 23.38 months in all patients, 22.91 months in the vertebroplasty group, 23.88 months in the unipedicular kyphoplasty group, and 23.44 months in the bipedicular kyphoplasty group. The mean follow-up was 42.2 months in all patients, 42.9 months in the vertebroplasty group, 41.8 months in the unipedicular kyphoplasty group, and 43.4 months in the bipedicular kyphoplasty group. There were no statistically significant differences between the three groups in terms of age, gender, t-score, mean injury time before the intervention and follow-up time.

OVCF were observed most commonly at the T12 and L1 level; the fracture ratio decreased thereafter as the levels extended to T1 to L5. These findings were statistically significant $(p<0.05)$ (Fig. 2).

In the vertebroplasty group, the mean VAS decreased from 8.3 preoperatively to 2.8 on the first day postoperatively, 2.96 at 6 months and 2.78 at 1 year. In the unipedicular kyphoplasty group, the mean VAS decreased from 8.3 preoperatively to 2.76 on the first day postoperatively, 2.85 at the sixth month and 2.84 at the first year. In the bipedicular kyphoplasty group, the mean VAS decreased from 8.27 preoperatively to 2.70 on the first day postoperatively, 2.79 at the sixth month and 2.66 at the first year. In the vertebroplasty group, the mean ODI

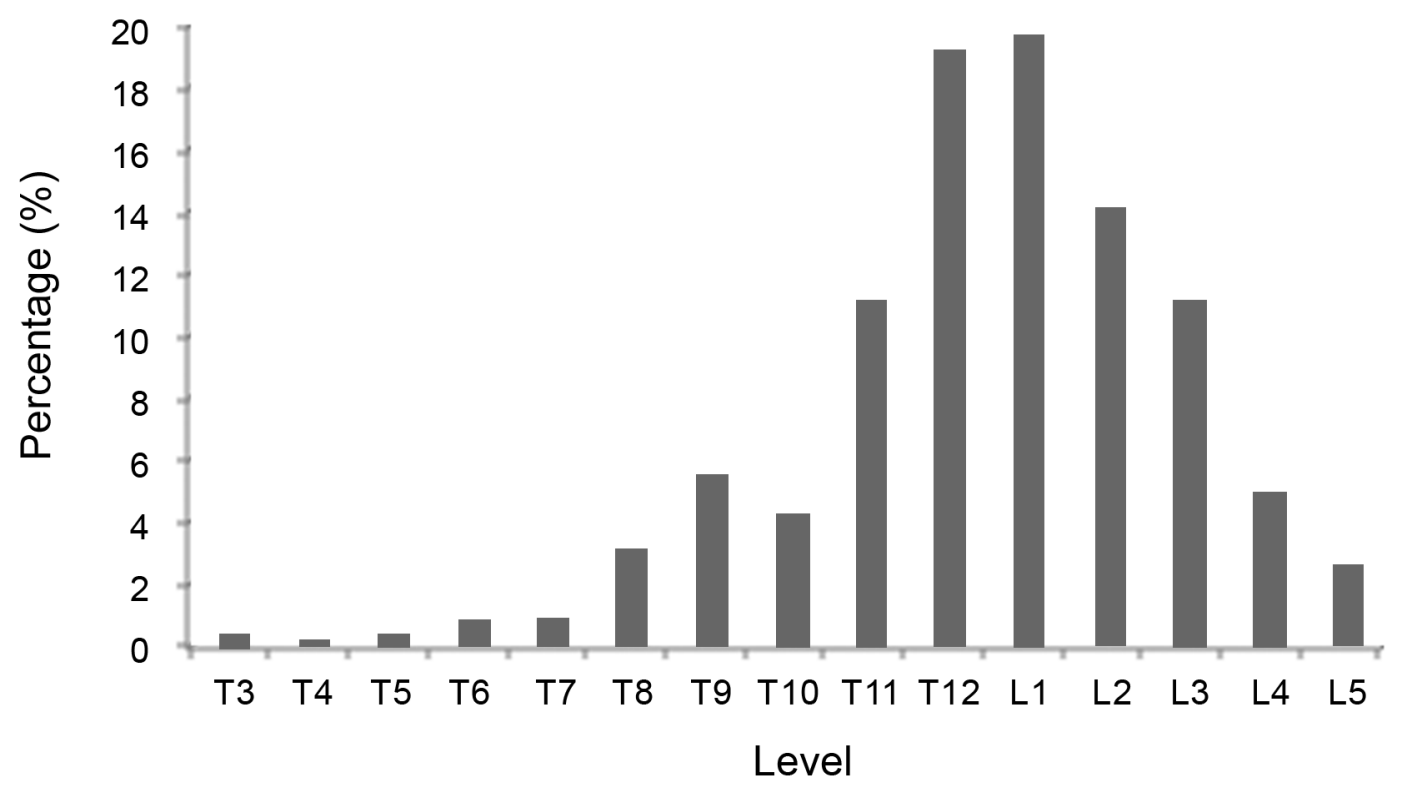

Fig. 2. Distribution of the affected levels. 
scores decreased from 74.42 preoperatively to 35.31 at the sixth month postoperatively and 26.56 at the first year. In the unipedicular kyphoplasty group, the mean ODI scores decreased from 73.45 preoperatively to 35.7 at 6 months postoperatively and 26.25 at 1 year. In the bipedicular kyphoplasty group, the mean ODI scores decreased from 73.80 preoperatively to 36.13 at the 6 months postoperatively and 26.01 at 1 year. The preoperative outcome scores between the three groups did not show any statistical difference. All three groups showed a statistically significant decrease in both VAS (first day
[ $p<0.001]$, sixth month and first year postoperatively) and ODI scores (sixth month and first year postoperatively, $p<0.001)$. There was no statistical difference between the unipedicular kyphoplasty, bipedicular kyphoplasty and vertebroplasty groups with regard to VAS values $(p>0.05)$. There was also no statistical difference between the unipedicular kyphoplasty, bipedicular kyphoplasty and vertebroplasty groups with regard to ODI scores $(p>0.05)$, (Figs. 3,4$)$. There were no gender differences in outcomes $(p>0.05)$.

The mean compression rate was $41 \%$ in all patients, $40 \%$

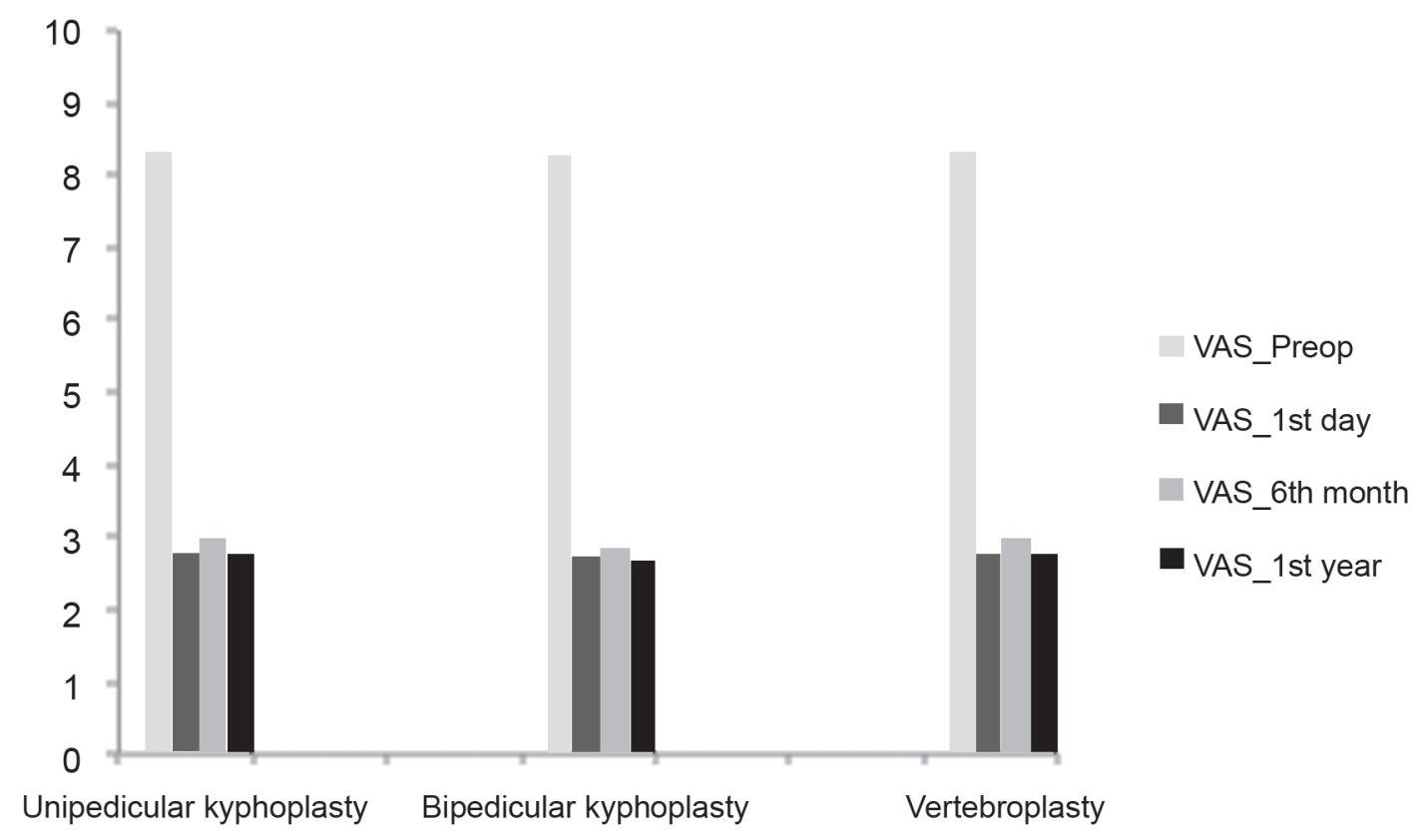

Fig. 3. Preoperative, first day, sixth month and one year visual analogue scale (VAS).

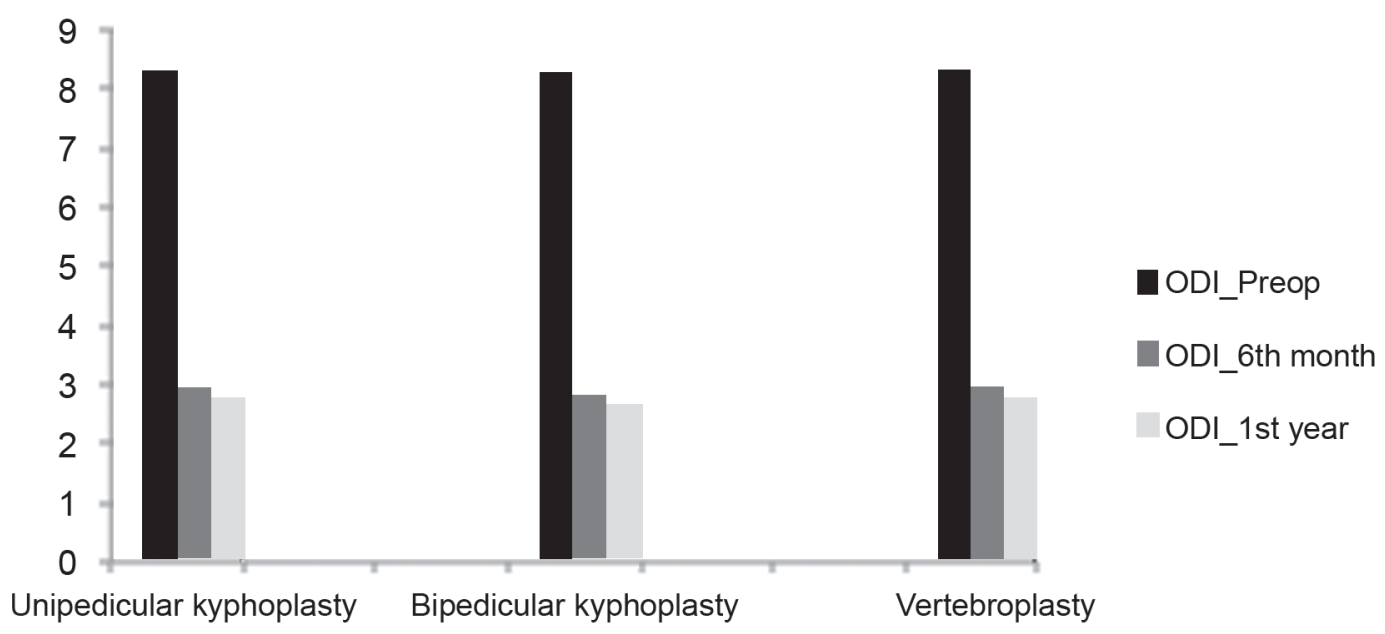

Fig. 4. Preoperative, first day, sixth month and one year Oswestry Disability Index (ODI) scores. 
in the vertebroplasty group $40 \%$ in the unipedicularkyphoplasty group, and $41 \%$ in the bipedicularkyphoplasty group. There was no statistical difference between the groups in terms of compression rate. The mean amount of cement injected was $2.9 \mathrm{~cm}^{3}$ (range, $1.0-6.2 \mathrm{~cm}^{3}$ ) in the vertebroplasty group, $1.5 \mathrm{~cm}^{3}$ (range, $0.75-3.1 \mathrm{~cm}^{3}$ ) in the unipedicular kyphoplasty group and $3.0 \mathrm{~cm}^{3}$ (range, $1.75-4.9 \mathrm{~cm}^{3}$ ) in the bipedicular kyphoplasty group. The mean height restoration rate was $24.16 \% \pm 1.27 \%$ in the vertebroplasty group, $24.25 \% \pm 1.28 \%$ in the unipedicular kyphoplasty group and $37.05 \% \pm 1.21 \%$ in the bipedicular kyphoplasty group. There was no statistical difference between the unipedicular kyphoplasty and vertebroplasty groups with regard to height restoration rates $(p>0.05)$. However, differences were statistically significant with regard to height restoration between the vertebroplasty and bipedicular kyphoplasty groups $(p<0.001)$ and between the unipedicular and bipedicular kyphoplasty groups $(p<0.001)$ (Fig. 5).

The majority of the complications in the procedures were due to cement extravasation. There were 11 cases of cement extravasation in the vertebroplasty group $9.0 \%$, 13 cases in the unipedicular kyphoplasty group $7.2 \%$ and 9 cases in the bipedicular kyphoplasty group $7.7 \%$. The cement extravasation was observed most commonly into the intradiscal space, and then anterior or lateral to the vertebral body. There was no cement leakage into the spinal canal or neural foramen in our cases. The cement extravasation rates between the three groups did not show any statistical difference. There were 4 cases of adjacent vertebral fracture in the vertebroplasty group (2.4\%), 4 cases in the unipedicular kyphoplasty group (3.4\%) and 2 cases in the bipedicular kyphoplasty group (2.3\%). There was no statistical difference between the groups in terms of adjacent vertebral fracture ( $p>0.05)$. No mortalities, infections or pulmonary embolisms were observed in our cases.

\section{Discussion}

Vertebral compression fractures are the most frequently observed complication and leading cause of morbidity and mortality of geriatric patients [4]. The fractures commonly occur in the mid-thoracic, low thoracic and high lumbar areas, and mostly at the thoracolumbar junction, especially L1 [13]. In our study, the levels most vulnerable to osteoporotic compression fractures were T12 and L1, and the differences were statistically significant.

Initial therapy for OVCF's are, sustained bracing and prolonged bed rest and pharmacologically with nonsteroidal anti-inflammatory drugs, narcotic medication and anti-resorptive therapy $[1,6]$. Some patients fail to benefit from these treatment modalities, and the diseaserelated morbidity persists. The pain and incapacity resulting from osteoporotic vertebral fractures demanded the development of new treatment modalities for effective therapy $[14,15]$.

Two related techniques were developed to regain vertebral body height and function and to decrease pain and incapacity: percutaneous vertebroplasty, in which cement is inserted into the vertebral body to reinforce the fractured bone; and kyphoplasty, a modification of vertebroplasty in which a balloon is inserted and inflated in a compressed vertebral body, reestablishing the compressed bone's height. Reported clinical studies concerning the outcomes demonstrated similar results in terms of pain and functional status improvement both for vertebroplasty and kyphoplasty [16-18]. Like previous studies both pain scores and functional outcome measurements showed significant improvement in our study. When

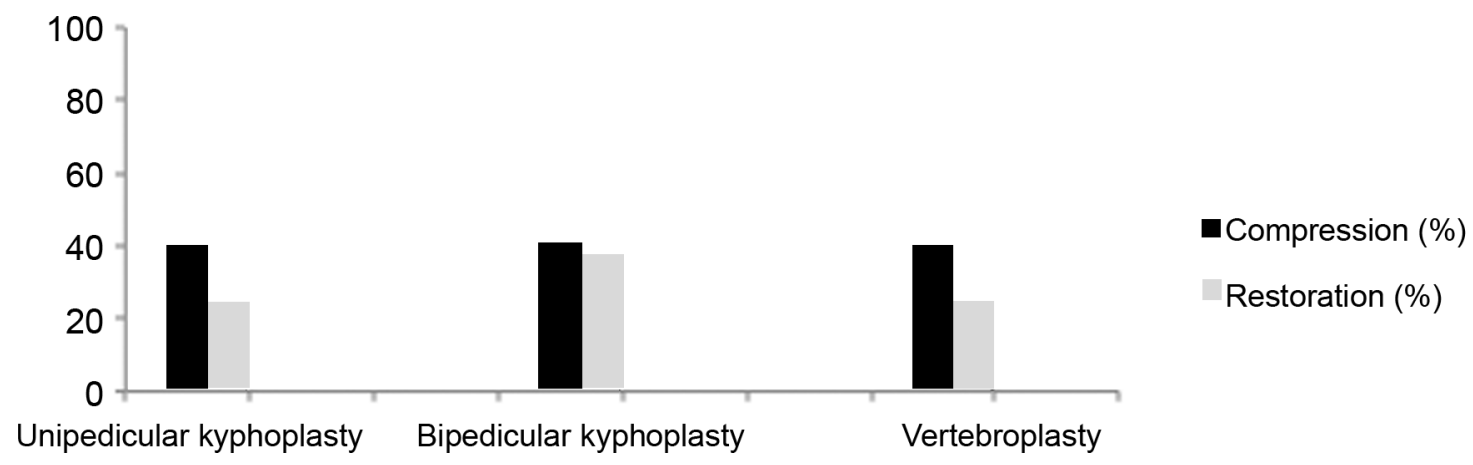

Fig. 5. Compression and height restoration rates. 
compared to conservative therapy, these procedures are effective, and long lasting with acceptable complication rates. While comparing these two procedures, the main controversy is not about efficacy and safety; it is about stating a specific indication for one over the other. Thus a competitive environment has risen between these procedures.

Although the biomechanical forces of compressed vertebrae sustain anterior collapse, we used anterior height for observing the height restoration rate after the intervention because of the undemanding prediction of preillness vertebral height. After establishing an unremarkable difference between the unipedicular and bipedicular approaches concerning height restoration rates in cadaveric studies, authors compared both unipedicular and bipedicular kyphoplasty for painful osteoporotic vertebral fractures, and reported that these two approaches result in the same acceptable clinical consequences despite the fact that bipedicular kyphoplasty is more efficacious in height restoration [19]. Some authors also reported that, vertebroplasty is also effective in terms of height restoration in selected patients $[20,21]$. Time of admission was stated as a prime determiner for height restoration. In our study, patients received interventions consecutively with the statistically same mean admission time. The bipedicular kyphoplasty group showed $37.5 \%$ height restoration and results were similar with previous studies $[9,19]$ but unipedicular kyphoplasty and the vertebroplasty group showed $24.25 \%$ and $24.16 \%$, respectively.

In terms of complications, both vertebroplasty and kyphoplasty are safe procedures. Cement leakage is the most commonly observed complications of these interventions. Recent studies showed approximately $10 \%$ cement leakage $[9,13,22]$. Similarly with these studies, the overall cement leakage rate was $7.6 \%$ and all were asymptomatic. This study confirms that both unipedicular and bipedicular kyphoplasty were related with the low rate of cement leakage compared to vertebroplasty, however, the difference was not statistically significant.

In our study, we compared the outcomes of unipedicular kyphoplasty, unipedicular vertebroplasty and bipedicular kyphoplasty in treating painful OVCF with regard to VAS, ODI scores and height restoration rates. Each group achieved satisfactory clinical outcomes according to VAS and ODI scores, and there were no statistical differences between the three groups after surgery. To our knowledge, no studies have clinically compared unipedicular kyphoplasty, unipedicular vertebroplasty and bipedicular kyphoplasty. Our results indicated that while all three groups achieved significant vertebral height restoration separately, bipedicular kyphoplasty is more successful in height restoration having a statistically significant difference in comparison with the other two groups.

\section{Conclusions}

Vertebroplasty and kyphoplasty, regardless of the bipedicular or unipedicular approach, are both effective in providing pain relief and improvement in functional capacity and quality of life after the procedure. The study concludes that, given the benefits of a unipedicular approach in connection with pedicle cannulation risk, surgery time, radiation exposure, and cost, unipedicular balloon kyphoplasty can be used as an alternative treatment for patients with OVCFs. Moreover, the bipedicular kyphoplasty procedure has a further advantage in terms of height restoration when compared to unipedicular kyphoplasty and vertebroplasty procedures.

\section{Conflict of Interest}

No potential conflict of interest relevant to this article was reported.

\section{References}

1. Riek AE, Towler DA. The pharmacological management of osteoporosis. Mo Med 2011;108:118-23.

2. Cummings-Vaughn LA, Gammack JK. Falls, osteoporosis, and hip fractures. Med Clin North Am 2011;95:495-506.

3. Bonnick SL. Osteoporosis in men and women. Clin Cornerstone 2006;8:28-39.

4. Dennison E, Cooper C. Epidemiology of osteoporotic fractures. Horm Res 2000;54 Suppl 1:58-63.

5. David C, Confavreux CB, Mehsen N, Paccou J, Leboime A, Legrand E. Severity of osteoporosis: what is the impact of co-morbidities? Joint Bone Spine 2010;77 Suppl 2:S103-6.

6. Rapado A. General management of vertebral fractures. Bone 1996;18:191S-6S.

7. Brown CJ, Friedkin RJ, Inouye SK. Prevalence and outcomes of low mobility in hospitalized older patients. J Am Geriatr Soc 2004;52:1263-70. 
8. Babayev M, Lachmann E, Nagler W. The controversy surrounding sacral insufficiency fractures: to ambulate or not to ambulate? Am J Phys Med Rehabil 2000;79:404-9.

9. Majd ME, Farley S, Holt RT. Preliminary outcomes and efficacy of the first 360 consecutive kyphoplasties for the treatment of painful osteoporotic vertebral compression fractures. Spine J 2005;5:244-55.

10. Fairbank JC, Pynsent PB. The Oswestry Disability Index. Spine (Phila Pa 1976) 2000;25:2940-52.

11. Sidney S, Castellan C. The case of $\mathrm{k}$ related samples, Section 7.2. In: Sidney S, Castellan C, editors. Nonparametric statistics for the behavioral sciences. 2nd ed. London: McGraw-Hill; 2005. p.174-83.

12. Brunner E, Domhof S, Langer F. Nonparametric analysis of longitudinal data in factorial experiments. New York Wiley; 2002.

13. Ledlie JT, Renfro M. Balloon kyphoplasty: one-year outcomes in vertebral body height restoration, chronic pain, and activity levels. J Neurosurg 2003;98:3642.

14. Wiwanitkit V. Unipedicular baloon kyphoplasty. Asian Spine J 2011;5:277.

15. An KC, Kang S, Choi JS, Seo JH. The clinical and radiological availability of percutaneous balloon kyphoplasty as a treatment for osteoporotic burst fractures. Asian Spine J 2008;2:9-14.
16. Grados F, Depriester C, Cayrolle G, Hardy N, Deramond $\mathrm{H}$, Fardellone $\mathrm{P}$. Long-term observations of vertebral osteoporotic fractures treated by percutaneous vertebroplasty. Rheumatology (Oxford) 2000;39:1410-4.

17. Diamond TH, Clark WA. Percutaneous vertebroplasty: a novel treatment for acute vertebral fractures. Med J Aust 2001;174:398-400.

18. Garfin SR, Yuan HA, Reiley MA. New technologies in spine: kyphoplasty and vertebroplasty for the treatment of painful osteoporotic compression fractures. Spine (Phila Pa 1976) 2001;26:1511-5.

19. Chen C, Chen L, Gu Y, et al. Kyphoplasty for chronic painful osteoporotic vertebral compression fractures via unipedicular versus bipedicular approachment: a comparative study in early stage. Injury 2010;41:3569.

20. Hiwatashi A, Moritani T, Numaguchi Y, Westesson PL. Increase in vertebral body height after vertebroplasty. AJNR Am J Neuroradiol 2003;24:185-9.

21. McKiernan F, Jensen R, Faciszewski T. The dynamic mobility of vertebral compression fractures. J Bone Miner Res 2003;18:24-9.

22. Lieberman IH, Dudeney S, Reinhardt MK, Bell G. Initial outcome and efficacy of "kyphoplasty" in the treatment of painful osteoporotic vertebral compression fractures. Spine (Phila Pa 1976) 2001;26:1631-8. 